\title{
Identify Shifts of Word Semantics through Bayesian Surprise
}

\author{
Zhuofeng $\mathrm{Wu}$ \\ Zhejiang University \\ wuzhuofeng@zju.edu.cn
}

\author{
Cheng Li \\ University of Michigan \\ lichengz@umich.edu
}

\author{
Zhe Zhao \\ University of Michigan \\ zhezhao@umich.edu
}

\author{
Fei $\mathrm{Wu}$ \\ Zhejiang University \\ wufei@cs.zju.edu.cn
}

\author{
Qiaozhu Mei \\ University of Michigan \\ qmei@umich.edu
}

\begin{abstract}
Much work has been done recently on learning word embeddings from large corpora, which attempts to find the coordinates of words in a static and high dimensional semantic space. In reality, such corpora often span a sufficiently long time period, during which the meanings of many words may have changed. The co-evolution of word meanings may also result in a distortion of the semantic space, making these static embeddings unable to accurately represent the dynamics of semantics. In this paper, we present a novel computational method to capture such changes and to model the evolution of word semantics.

Distinct from existing approaches that learn word embeddings independently from time periods and then align them, our method explicitly establishes the stable topological structure of word semantics and identifies the surprising changes in the semantic space over time through a principled statistical method. Empirical experiments on large-scale real-world corpora demonstrate the effectiveness of the proposed approach, which outperforms the state-of-the-art by a large margin.
\end{abstract}

\section{CCS CONCEPTS}

- Computing methodologies $\rightarrow$ Lexical semantics; • Information systems $\rightarrow$ Language models; Similarity measures;

\section{KEYWORDS}

Word Embeddings; Semantic Shifts; Bayesian Surprise

\section{ACM Reference Format:}

Zhuofeng Wu, Cheng Li, Zhe Zhao, Fei Wu, and Qiaozhu Mei. 2018. Identify Shifts of Word Semantics through Bayesian Surprise. In SIGIR '18: The 41st International ACM SIGIR Conference on Research Development in Information Retrieval, fuly 8-12, 2018, Ann Arbor, MI, USA. ACM, New York, NY, USA, 10 pages. https://doi.org/10.1145/3209978.3210040

\footnotetext{
Permission to make digital or hard copies of all or part of this work for personal or classroom use is granted without fee provided that copies are not made or distributed for profit or commercial advantage and that copies bear this notice and the full citation on the first page. Copyrights for components of this work owned by others than ACM must be honored. Abstracting with credit is permitted. To copy otherwise, or republish, to post on servers or to redistribute to lists, requires prior specific permission and/or a fee. Request permissions from permissions@acm.org.

SIGIR '18, fuly 8-12, 2018, Ann Arbor, MI, USA

(C) 2018 Association for Computing Machinery.

ACM ISBN 978-1-4503-5657-2/18/07 ..\$15.00

https://doi.org/10.1145/3209978.3210040
}

\section{INTRODUCTION}

${ }^{1}$ Language is a mirror of reality ([26], p. 6): environment, society, culture, technology. When reality changes, language changes. Reality is a function of language [15]. Language influences our view of the world and influences social structures. Failing to adjust to the changes of language, fails to adapt to the reality.

If Vannevar Bush were still alive and read this paper, he should be surprised about how differently the word "retrieval" means compared to his time. Would he still name his imaginary machine "memex" if he knew the modern meanings of "memory?" Consider Marty McFly coming to the future and searching for "an apple store"; consider him asking about "weeds" on Reddit; consider inviting a 13th-century knight and a 16th-century scholar to a "bachelor" party. Wait, did you just say "party?"

Thanks to the fast evolution of technology and the prevalence of social media, meanings of words have been changing more and more rapidly. What is your first impression of "deep?" "CNN?" "At?" How to detect and adapt to these changes is a critical challenge of natural language processing, which largely affects the performance of downstream tasks such as Web search, information filtering, personalized recommendation, and dialog system.

Measuring frequency is easy; measuring meanings is hard. Many existing studies have been done to analyze the change of semantics (i.e., meanings) of certain words (e.g., [5, 19, 39]), but the need of careful linguistic investigations makes them hard to scale. Recent successes of large-scale word representation learning have inspired researchers to rethink about detecting semantic shifts. Comparing to traditional methods based on qualitative analysis, these methods can efficiently compute the embeddings of words in vector spaces, so that their meanings can be analyzed in a quantitative way.

Multiple methods have been proposed recently that build upon word embedding methods (such as the Word2Vec [30]). Instead of focusing on a small number of target words, these methods massively compute and rank the differences of the embeddings of many words across different time periods (e.g., [14, 20, 21]). Words whose embedding vectors diverge significantly are suspected to have a change of semantics. As a result, these methods do retrieve the words that have truly changed their meanings, but they also introduce a large number of false positives. Extensive manual work has to be done to scan through the words that have passed the filter in order to identify the true changes.

How to identify shifts of word semantics with both a high recall and a high precision? First, to answer the question what has changed, it is important to consider what is consistent. In fact, one

\footnotetext{
${ }^{1}$ This work was done when the first author was visiting the University of Michigan.
} 
critical problem of the existing approaches is the lack of a fixed frame of reference, in particular, a semantic space that is reasonably stable over time so that the shifts of words in that space can be detected. If the semantic space itself changes, so do most of the words in the space. As a result, many "semantic" shifts identified by existing methods are artifacts of the distortion of the space. Second, to claim that a word's meaning has changed, one has to be certain about its old meanings. In other words, to identify true shifts of semantics, one should to be able to distinguish real changes from noises. If there is a high uncertainty about a word in the semantic space, a "shift" might have been a random walk.

In this paper, we present a novel design of methods to accurately identify shifts of word semantics, which successfully addresses the two critical problems above. Instead of attempting to align the word embeddings to obtain the same frame of reference, we represent word semantics in a topological space that is robust to distortions over time. We propose a novel and effective method to detect shifts in this space, which is based on a principled statistical model called the Bayesian surprise. The method can successfully distinguish true changes of word semantics from noises and uncertainty, which significantly increases the precision without sacrificing the recall. We present carefully designed experiments on four large scale, real world data sets. The proposed method is evaluated in two modes, to detect synthetic changes and real changes, respectively. It achieves near-perfect performance on detecting synthetic changes, outperforming all competing methods by large margins, as well as clearly better performance on detecting real changes.

\section{RELATED WORK}

The key innovation of our work is to compute a topological representation of the semantic space and to identify shifts in that space through Bayesian surprise. There are four lines of literature that are closely related to our work: word embeddings, semantic shift detection, Bayesian surprise and dynamic topic models.

\subsection{Word Embeddings}

Word embeddings essentially compute the coordinates of words in a low-dimensional vector space. Bengio et al. [4] proposed a neural language model, which is optimized through stochastic gradient descent and outperforms traditional n-gram models. Mikolov et al. $[29,30]$ proposed Word2Vec, which adopts optimization methods such as negative sampling and abstract stochastic gradient descent [35]. Their model greatly reduces the training time and effectively improves the quality of the embedding vectors. Tang et al. [37] proposed the LINE, a network embedding method that is able to preserve both the local and global network structures. When applied to a word co-occurrence network, network embedding methods such as LINE and DeepWalk [33] learn word embeddings.

A recent study by Levy et al. [24] reveals that the performance gain of word embeddings are due to certain system design choices and hyper-parameter optimization. The essence of word embeddings is related to the Singular Value Decomposition (SVD) of the pointwise mutual information (PMI) matrix [9].

The first step of our method learns word embeddings from individual time periods, and any reasonable word embedding method can be adopted for this purpose.

\subsection{Semantic Shift Detection}

There have been several studies on detecting semantic changes over time. Most of them learn separate embeddings for different time periods and identify the changes by comparing these embeddings.

In order to compare word vectors from different periods, one has to ensure that the vectors are aligned into one unified coordinate system. Kim et al. [20] adopted the Skip-gram model [29, 30] to train embeddings from individual time periods, and they initialized the word vectors in subsequent years with the word vectors obtained from the previous years. They identified semantic shifts by comparing the cosine similarity of the embedding vectors for the same words in different years. Kulkarni et al. [21] addressed the embedding alignment problem by learning an alignment function, through a Linear Regression (LR) or a Local Linear Regression (LLR). Hamilton et al. [14] proposed a slightly different approach. They used orthogonal Procrustes to align the embeddings among different periods, which preserves the cosine similarities by the largest extent. Different from these studies, we represent word semantics in a topological space instead of a vector space, which is robust to distortions and avoids the hassle of aligning embeddings.

Other work also tried to avoid the alignment problem in various ways. Gulordava and Baroni [12] used the cosine product of context vectors to measure the distributional similarity of words in the Google Books N-gram Corpus between two periods. Brigadir et al. [7] compared the nearest neighbors of a word from different time periods and scored semantic shifts using Jaccard distance. Similarly, Hamilton et al. [13] defined semantic changes by measuring local neighborhoods. They compared the nearest neighbors from three periods and defined a cosine-similarity function between two sets. Our work differs from the above studies by considering all historical periods to construct and update the topological semantic space and to quantify semantic shifts.

Frermann and Lapata proposed a dynamic Bayesian model [10] to track the change of word meanings. They represented a word by a set of senses, with each sense being a probability distribution over words, through which one can directly observe the change of these senses. Such an approach makes a strong assumption on the generation of word senses, and it cannot quantify the changes of the induced senses. In our work, we explicitly quantify the surprise introduced by the new data, without making assumptions on the numbers and distributions of word senses.

Comparing to all existing work on semantic shift detection, the biggest difference of our method is the use of Bayesian surprise to formally model and quantify shifts in a topological semantic space.

\subsection{Bayesian Surprise}

Bayesian surprise is a formal statistical method proposed by Itti and Baldi [17], which explicitly measures the influence of a new data point on the background data. Studies show that Bayesian surprise well predicts human attentions [17]. Bayesian surprise has been successfully applied to various tasks, such as computer vision [11], neuro-imaging [31], and landmark detection [34].

Bayesian surprise was first applied to text analysis by Louis [25], for the task of text summarization. She used Bayesian surprise to compute a surprise value for each word type in the input and then extracted the sentences with highest surprise values. 
We present the first work that applies Bayesian surprise to quantify the shifts of word meanings.

\subsection{Dynamic Topic Models}

To model the evolution and dynamics of latent topics, Mei and Zhai extracted topics from individual time periods and connected similar topics in different periods [27]. Blei and Lafferty proposed the Dynamic Topic Model (DTM) [6] which models the evolution of topic-word distributions over time. Wang and McCallum [40] adopted a different angle to model topic trends. They treated the word co-occurrences and documents' time stamps as observations of the latent topics. None of these methods quantifies the change of individual terms and thus cannot be applied to our problem.

\section{PROBLEM FORMULATION}

We aim to detect words that present significant changes in their meanings within a certain time period (or an epoch). Let us consider a longitudinal corpus $C$ that is divided into $n$ epochs $\left\{C_{t}\right\}$, where $t \in\{1,2, \ldots, n\}$ indicates a particular time period. Let $\Phi_{t}(V)$ denote the representation of the semantics of all words in the vocabulary $V$ at time $t$, and $\phi_{t}(v)$ the representation of the meaning of word $v \in V$. We are interested in measuring the deviation of meaning of $v$ during the period $t$, that is, $\Delta\left(\phi_{t-1}(v), \phi_{t}(v)\right)$. Words with a high $\Delta\left(\phi_{t-1}(v), \phi_{t}(v)\right)$ are considered as shifted during time period $t$.

In general, assuming that most words do not have a meaning change in a single epoch, $\Delta\left(\phi_{t-1}(v), \phi_{t}(v)\right)$ should be close to zero in most cases. The fundamental question here is how to establish a good representation of $\phi_{t}(v)$ and how to make $\phi_{t}(v)$ and $\phi_{t-1}(v)$ comparable so that $\Delta\left(\phi_{t-1}(v), \phi_{t}(v)\right)$ can be calculated. In practice, many existing approaches tend to model $\phi_{t}(v)$ as a vector in a high-dimensional space of word semantics (i.e., $\Phi_{t}(V)$ ) which is learned from $C_{t}$ through word embedding methods such as Word2Vec. All these approaches face two critical problems: 1) the word embedding $\phi_{t}(v)$ learned from a single epoch of data $C_{t}$ might not sufficiently represent the meanings of $v$, and 2) the semantic spaces learned independently from two epochs, e.g., $\Phi_{t-1}(V)$ and $\Phi_{t}(V)$, might not align accurately. The first problem makes the computed $\Delta\left(\phi_{t-1}(v), \phi_{t}(v)\right)$ vulnerable to biases and random noises, and the second problem leads to many false positives as a high $\Delta\left(\phi_{t-1}(v), \phi_{t}(v)\right)$ may be an artifact of the misalignment of the semantic spaces. As a result, many heuristics have to be used to align/augment the embeddings learned from individual epochs.

Without loss of generality, we may consider $\Phi_{t}(V)$ as a function of $\left\{C_{1}, C_{2}, \ldots, C_{t}\right\}$ instead of just $C_{t}$, and $\phi_{t}(v)$ as a function of $\Phi_{t}(V)$ instead of just $v$. That says, the semantic space of language does not only depend on the current time period but should also reflect the historical usage of words; and the meaning of one word is not simply indicated by its own position in the space but should also depend on how it relates to other words.

This general formulation enables us to formally consider the relativity in the evolution of a semantic space over time, namely: what remains still and what moves? Indeed, if word semantics is represented with a vector space, it is critical to make sure that there is no distortion of its dimensions over time, so that words whose meanings did not change will remain in the same positions. Moreover, if the meaning of a word has truly shifted, that change should be robust to the noises in the semantic space, noises that are either introduced due to the sparsity of the observations or due to the uncertainty of the meanings.

To model the stability and the changes of word semantics, we focus on a topological representation of the semantic space (instead of a vector space), which is robust to distortions over time. Changes of the topology are identified through a principled statistical method, the Bayesian surprise, so that they are robust to uncertainty and random noises. The proposed method will be described in details in the following section.

\section{METHOD TO DETECT SEMANTIC SHIFTS}

We propose a carefully designed method that establishes the topological representations of word semantics over time and uses Bayesian surprise to identify significant shifts of word semantics.

\subsection{Design Rationale}

Our method builds upon the insight that a topological structure is an effective representation of the stability of the semantic space and Bayesian surprise is a powerful tool to identify significant changes. Shifts identified through Bayesian surprise in a topological semantic space are robust to distortions and noises introduced by the partition of the corpus into multiple epochs. To better understand the rationale of our design, it is useful to think about the limitations of alternative approaches:

- Word embeddings obtain vector representations of word semantics. If the coordinates of a word in the learned vector space changes, the meaning of the word may have changed. However, a change of the absolute coordinates of a word isn't necessarily caused by a shift of meaning. Any distortion of the vector space, such as a rotation, a permutation, a change (increase, decrease) of dimensionality, a merger or a split of certain dimensions, would have resulted in an apparent change of the coordinates of the same word. Therefore, heuristics are used to control the distortion of the vector spaces over time, such as post hoc alignment of two vector spaces, using one embedding to initialize/regularize the learning of the next embedding, imposing strong priors of how the vector spaces evolve, etc. These heuristics can control the distortions to some extent, but their effectiveness in practice is usually limited. For example, it is hard to establish a perfect alignment of two embeddings, and even a small misalignment would accumulate over multiple epochs. A topological space has a fundamental advantage over vector spaces, as it is robust to such distortions over time.

- Unlike word embeddings, a topological space does not define the semantics of a word by numerical coordinates of its position, but by its relative position to other words. If we represent semantics with a manifold (a special type of topological space), the meaning of a word can be interpreted by the nearest neighbors of the word in that space. While embedding vectors of words are vulnerable to noises and distortions, their local and global topology tend to sustain.

- We found that almost all existing approaches identify word semantic shifts through comparing only two consecutive epochs, which often suffers from a high risk of noise and 
uncertainty. We specifically adopt Bayesian surprise in our design to distinguish systematic shifts from noises and insufficient observations.

\subsection{Method Overview}

Our proposed method for detecting shifts of word semantics consists of three components: embedding learning, manifold construction, and change detection.

Like existing approaches, we first divide the corpus into subcorpora according to the time period each document was published in. Once the partition is done, we train word embeddings on each sub-corpus independently.

Unlike existing approaches, once the embeddings are trained, we do not attempt to compare them or align them. Instead we construct a topological semantic space (i.e., a semantic manifold) based on these embeddings.

Whenever a new time period is processed, the neighborhood of each word in the semantic manifold is updated: new neighbors added, existing neighbors reinforced, and outdated neighbors weakened.

By comparing the neighborhood of a word before and after the update, the change of its semantics can be measured. Note that this approach is different from comparing the two consecutive time periods, and all previous time periods will be taken into consideration. To stand out from this measurement, the neighborhood of the word must have sufficient observations in history and must present a surprising change in the current period. We use the Bayesian Surprise [16], a formal method that measures the Bayesian definition of surprise, to achieve this goal. Bayesian surprise provides an intuitive way to quantify the significance of shifts of a word's meaning with respect to its historical meanings.

\subsection{Learning Word Embeddings}

We adopt a commonly used embedding method called LINE [37]. LINE learns node representations of a network by optimizing an objective function that attempts to preserve both the first-order proximity and the second-order proximity. When applied to a simple word co-occurrence network where nodes are words and edges are weighted by the numbers of times two words co-occur in the same documents, LINE outputs the embedding of words.

The first-order proximity is determined by the co-occurrences of two words in the same context. The second-order proximity brings together two words that both co-occur with the same set of words, even though they themselves might not have co-occurred in the same context. LINE learns two types of embeddings separately by preserving either the first- or the second-order proximity, and the two embeddings are finally concatenated to form the final representation of each word.

In theory, any reasonable word embedding method can be used here. We select LINE as it does present advantages over some other methods such as the Skip-gram [30], due to the specific treatments to two types of proximities [37]. Readers are encouraged to explore other embedding methods and replicate our results.

\subsection{Constructing Semantic Manifold}

Having trained word embeddings $\Phi_{t}(V)$ for each time period $C_{t}$, we can construct and update the topological space of word semantics. Unless specifically regularized or aligned, the embeddings learned from different epochs are unlikely to share exactly the same dimensions. The topological space instead is likely to remain the same. That is, if two words were close (in meaning) in the semantic space of $t$ and their meanings did not change in $t+1$, they should still be close in the semantic space of $t+1$.

While other types of topological spaces can be used here too, we decide to construct a manifold of word semantics, or a semantic manifold. A manifold is a specialized topological space where the neighborhood of each point resembles a Euclidean space [23]. This is a neat property for our purpose, as we can use the Euclidean distance of the embedding vectors of two words to determine whether they should be in the neighborhood of a manifold.

Following the common practice of manifold learning [23], we approximate a semantic manifold by constructing a K-NearestNeighbor (KNN) graph, $G=(V, E)$, where $V$ is the set of words and $E$ is the set of directed edges between the words, with each edge $e=(u, v), e \in E$ indicating that word $v$ is among the top $K$ words with the closest semantics to word $u$ in at least one time period. We define the k-nearest-neighbors of a word $v$ at epoch $t$ in embedding space as: $k N N\left(\phi_{t}(v)\right)$. Then the nearest semantic networks at epoch $t$ can be constructed as follows:

$$
E_{t}=\left\{(u, v), \forall v \in k N N\left(\phi_{t}(u)\right), \forall u \in V_{t}\right\}
$$

Precisely calculating k-nearest neighbors for each words has a complexity of $O\left(|V|^{2} p\right)$ ( $p$ as the dimensionality of word embeddings), which is extremely expensive when the vocabulary size $|V|$ is colossal. We use the algorithm LargeVis ${ }^{2}$ from Tang et al. [36] to compute an approximate $\mathrm{KNN}$ graph. Similar to the definition of $\mathrm{k}$-nearest neighbors, we define the approximate nearest neighbors of a word $v$ at epoch $t$ in embedding space as: $a N N\left(\phi_{t}(v)\right)$.

Starting from the first time period, the semantic manifold is updated by augmenting $V_{t}$ and $E_{t}$ into the current graph $G$. That is, $V=V \cup V_{t}$ and $E=E \cup E_{t}$. Note that in a semantic manifold, the distance between two neighbors should be updated over time. That says, in the corresponding semantic graph, the weights of edges should also be updated. In practice, the edge weights could be updated based on certain functions of the actual Euclidean distance of the embedding vectors. For simplicity, in this paper we just augment the weight of $(u, v)$ by one if word $v$ appears in the neighborhood of $u$ in one more time period.

\subsection{Detecting Shift with Bayesian Surprise}

The semantic manifold constructed and updated over time represents the stability of the semantic space, which is robust to the misalignment and distortion of word embeddings learned from individual epochs. With the semantic manifold, identifying changes of word meanings becomes much easier. Intuitively, one may simply compare the neighbors of a word in the current epoch $\left(a N N\left(\phi_{t}(v)\right)\right)$ with the neighbors of $v$ in the semantic manifold as of $t-1$. Indeed, this can be done simply by comparing the two sets, e.g., through the Jaccard distance. However, this simple approach, although better

\footnotetext{
${ }^{2}$ https://github.com/lferry007/LargeVis
} 
than directly calculating the difference of two embedding vectors, may still suffer from two problems: 1) the observations of a word are not sufficient to support a systematic shift of its meaning; 2) there is a high variance of the neighbors among the past time periods.

Indeed, when a word is not frequently used or when it is very new, even though the nearest neighbors may have diverged a lot, the difference may have been an artifact of data sparsity and there is no sufficient evidence of a semantic shift. Similarly, if a word does not have a stable set of neighbors among past time periods, it indicates the meaning of that word has been uncertain, and a new set of neighbors should not be considered as a semantic shift. Both problems call for a method that accounts for the statistical significance of a change, which is beyond the capability of calculating the difference of two sets or two vectors. We address this problem with a principled statistical method, the Bayesian surprise. Below we first formally define Bayesian surprise, as proposed by Itti and Baldi [16]. Then we present how to use Bayesian Surprise to detect semantic changes of words.

Bayesian Surprise: According to the Bayesian Theorem [1], an observer has a prior probability $P(H)$ associated with each hypothesis $H \in \mathcal{H}$, where $\mathcal{H}$ is the space of all hypotheses based on the background knowledge of the observer. Given newly observed data (in our case, the sub-corpus of a new epoch) $C_{t+1}$, the posterior probability of a single hypothesis can be defined according to Bayesian Theorem: $P\left(H \mid C_{t+1}\right)=\frac{P\left(C_{t+1} \mid H\right) P(H)}{P\left(C_{t+1}\right)}$. The surprise carried by the new observation $C_{t+1}$ to the hypothesis space $\mathcal{H}$ is defined as the difference between the prior distribution and posterior distribution of the hypotheses. Naturally, we use the Kullback-Leibler $(K L)$ divergence [22] to compute the difference between two distributions:

$$
\begin{aligned}
S\left(C_{t+1}, \mathcal{H}\right) & =K L\left(P\left(H \mid C_{t+1}\right), P(H)\right) \\
& =\int_{\mathcal{H}} P\left(H \mid C_{t+1}\right) \log \frac{P\left(H \mid C_{t+1}\right)}{P(H)} .
\end{aligned}
$$

In our setting, we denote $\mathcal{H}_{v}$ as the set of all the hypotheses encoding background information of word $v$. We want to calculate the amount of surprise that is attached to the word $v$ when a new epoch's data $C_{t+1}$ arrives. In particular, we use a multinomial distribution to model the likelihood $P\left(C_{t+1} \mid H_{v}\right)$. We adopt Dirichlet distribution as our prior distribution, as it is the conjugate prior of the multinomial distribution. Denote Dirichlet distribution as $\operatorname{Dir}(x ; \alpha)$, where $\alpha=\left[\alpha_{1}, \alpha_{2}, \ldots, \alpha_{|V|}\right],|V|$ is the size of vocabulary, $\alpha_{u \in V}$ counts the appearances of word $u$ in the neighborhood of word $v$ in history (i.e., $\alpha_{u}=\left|\left\{t^{\prime} \in[t] \mid u \in \operatorname{aNN}\left(\phi_{t^{\prime}}(v)\right)\right\}\right|,[t]=\{1, \ldots, t\}$ ). Formally,

$$
\begin{aligned}
P\left(H_{v}\right) & =\operatorname{Dir}\left(x ; \alpha_{1}, \alpha_{2}, \ldots, \alpha_{|V|}\right) \\
& =\frac{\Gamma\left(\sum_{i=1}^{|V|} \alpha_{i}\right)}{\prod_{i=1}^{|V|} \Gamma\left(\alpha_{i}\right)} \prod_{i=1}^{|V|} x_{i}^{\alpha_{i}-1} .
\end{aligned}
$$

When the data of the new epoch $C_{t+1}$ (or new observations) is given, we can construct a new $\mathrm{KNN}$ graph $G_{t+1}$. Let $E_{t+1}(v)$ be the set of edges starting from word $v$ in the network $G_{t+1}$. For word $u \in V$, we define $\beta_{u}$ as the denotation of whether word $u$ appears in the nearest neighbor set of word $v$ in $G_{t+1}$ :

$$
\beta_{u}= \begin{cases}1, & (v, u) \in E_{t+1}(v) \\ 0, & \text { else }\end{cases}
$$

Then we can obtain the posterior distribution given by new observation $C_{t+1}$ on word $v$ :

$$
P\left(H_{v} \mid C_{t+1}\right)=\operatorname{Dir}\left(x ; \alpha_{1}+\beta_{1}, \alpha_{2}+\beta_{2}, \ldots, \alpha_{|V|}+\beta_{|V|}\right) .
$$

At last, we are able to calculate the surprise score on word $v$ as:

$$
\operatorname{Score}(v)=S\left(C_{t+1}, H_{v}\right)=K L\left(P\left(H_{v} \mid C_{t+1}\right), P\left(H_{v}\right)\right)
$$

When calculating the KL divergence, we use add-one smoothing for the Dirichlet distributions, which means the initial value of $\alpha_{u}$ is 1 at time zero. In practice, we follow settings given by Itti and Baldi [3], as well as the formulas from [32] to calculate the Bayesian surprise between two Dirichlet distributions. That is,

$$
\begin{aligned}
\operatorname{Score}(v) & =\int_{\mathcal{H}_{v}} P\left(H_{v} \mid C_{t+1}\right) \log \frac{P\left(H_{v} \mid C_{t+1}\right)}{P\left(H_{v}\right)} \\
& =\int \operatorname{Dir}(x, \alpha+\beta) \log \frac{\operatorname{Dir}(x, \alpha+\beta)}{\operatorname{Dir}(x, \alpha)} d x \\
& =\int(\log \operatorname{Dir}(x, \alpha+\beta)-\log \operatorname{Dir}(x, \alpha)) \operatorname{Dir}(x, \alpha+\beta) d x \\
& =\int\left(\log \Gamma\left(\alpha_{0}+\beta_{0}\right)-\sum_{i=1}^{|V|} \log \Gamma\left(\alpha_{i}+\beta_{i}\right)\right. \\
& +\sum_{i}\left(\alpha_{i}+\beta_{i}-1\right) \log x_{i}-\log \Gamma\left(\alpha_{0}\right) \\
& \left.+\sum_{i=1}^{|V|} \log \Gamma\left(\alpha_{i}\right)-\sum\left(\beta_{i}-1\right) \log x_{i}\right) \operatorname{Dir}(x, \alpha+\beta) d x
\end{aligned}
$$

where $\alpha_{0}=\sum_{i=1}^{|V|} \alpha_{i}$ and $\beta_{0}=\sum_{i=1}^{|V|} \beta_{i}$.

Because $\operatorname{Dir}(x, \alpha+\beta)$ is a probability function, we have: $\int \operatorname{Dir}(x, \alpha+$ $\beta) d x=1$. Besides, the geometric mean $\int \operatorname{Dir}(x, \alpha+\beta) \log x_{i} d x$ equals $\psi\left(\alpha_{i}+\beta_{i}\right)-\psi\left(\alpha_{0}+\beta_{0}\right)$, where $\psi$ is the digamma function. Then we get:

$\operatorname{Score}(v)=\log \frac{\Gamma\left(\alpha_{0}+\beta_{0}\right)}{\Gamma\left(\alpha_{0}\right)}+\sum_{i=1}^{|V|} \log \frac{\Gamma\left(\alpha_{i}\right)}{\Gamma\left(\alpha_{i}+\beta_{i}\right)}+\sum_{i=1}^{|V|} \beta_{i}\left(\psi\left(\alpha_{i}\right)-\psi\left(\alpha_{0}\right)\right)$

We can further simplify this equation as follows to avoid direct calculation of $\Gamma(x)$ :

$\operatorname{Score}(v)=\sum_{j=\alpha_{0}}^{\alpha_{0}+\beta_{0}-1} \log j-\sum_{i=1}^{|V|} \sum_{j=\alpha_{i}}^{\alpha_{i}+\beta_{i}-1} \log j+\sum_{i=1}^{|V|} \beta_{i}\left(\psi\left(\alpha_{i}\right)-\psi\left(\alpha_{0}\right)\right)$

Words with a high score by Equation 9 are considered to be shifted in semantics during the current time period.

\section{EXPERIMENTS}

In this section, we present empirical experiments that compare the proposed method with various baselines and alternative methods on four public data sets.

\subsection{Competing Methods}

We compare the proposed method against three types of baselines, two representing how the task is done in previous studies, 
by computing the changes of the embeddings of words, and one representing an alternative version of the proposed method. They include (i) a method [20] that uses the embeddings trained in previous time period as the initialization of the current period, (ii) a method [21] that independently learns embeddings from different periods and aligns them thereafter, and (iii) a method that uses the Jaccard Distance to quantify the topological structure change.

Init: this method uses the previous epoch's embedding as the initialization for the current epoch's embedding, then trains the embeddings with LINE [37], and then uses the Euclidean distance between the embedding vectors of a word in two epochs to quantify the change, that is, $\operatorname{Score}(v)=\left\|\phi_{t+1}(v)-\phi_{t}(v)\right\|$. In practice, we have explored both the Euclidean distance and the Cosine similarity as the scoring function and did not observe much difference. We therefore use the Euclidean distance for all methods.

LR: this method trains word embeddings independently for each time period, and then aligns them through Linear Regression [21]. An important prerequisite for this method is that most words did not change. Given this prerequisite, one can align the embedding spaces into one unified coordinate system and characterize the change of meanings. Suppose that we have trained two embeddings $\Phi_{t}(V)$ and $\Phi_{t+1}(V)$, we seek to learn a linear transformation $\mathbf{L} \mathbf{R}_{t \rightarrow t+1}(v) \in$ $\boldsymbol{R}^{p \times p}$ that maps a word $v$ from $\phi_{t}(v)$ to $\phi_{t+1}(v)$ by solving the following optimization problem:

$$
\underset{t \rightarrow t+1}{\mathbf{L R}}=\underset{\mathbf{L R}}{\operatorname{argmin}} \sum_{v \in V}\left\|\phi_{t}(v) \mathbf{L R}-\phi_{t+1}(v)\right\|_{2}^{2} .
$$

Denote $\phi_{t}^{\prime}(v)=\phi_{t}(v) \mathbf{L} \mathbf{R}_{t \rightarrow t+1}(v)$ the transformation of $\phi_{t}(v)$. For some words, the difference between $\phi_{t}^{\prime}(v)$ and $\phi_{t+1}(v)$ could be very large, and it is likely that the meanings of these words have shifted. So we can define the change as:

$$
\operatorname{Score}(v)=\left\|\phi_{t}(v) \mathbf{L R}_{t \rightarrow t+1}(v)-\phi_{t+1}(v)\right\| .
$$

LLR: this method is similar to LR, but uses Local Linear Regression as its alignment function [21]. Unlike $L R$ that learns a transformation function using all words, $L L R$ only considers nearest neighbors. Denote the k-nearest neighbors of a word $v$ at epoch $t$ in embedding space as: $k N N\left(\phi_{t}(v)\right)$. Having trained two embeddings $\Phi_{t}(V)$ and $\Phi_{t+1}(V)$, we seek to learn a local linear transformation $\operatorname{LLR}_{t \rightarrow t+1}(v) \in R^{p \times p}$ that maps a word $v$ from $\phi_{t}(v)$ to $\phi_{t+1}(v)$ by solving the following optimization problem:

$$
\underset{t \rightarrow t+1}{\mathbf{L L R}(v)}=\underset{\mathbf{L L R}}{\operatorname{argmin}} \sum_{w \in k N N\left(\phi_{t}(v)\right)}\left\|\phi_{t}(w) \mathbf{L L R}-\phi_{t+1}(w)\right\|_{2}^{2}
$$

Then the displacement is defined as:

$$
\operatorname{Score}(v)=\left\|\phi_{t}(v) \mathbf{L L R}(\mathbf{v})-\phi_{t+1}(v)\right\| .
$$

Apparently, all Init, LR, and LLR make the assumption that the dimensionality of the semantic space is a constant over time, which might not hold in reality. By comparing to these methods, we can understand the benefits of representing the semantics space as a manifold instead of a vector space.

aNN+Jaccard: this method uses a topological representation of the semantics space and uses the Jaccard distance [2] to identify the change, which is similar to the method used in $[7,13]$. Like the proposed method, it first uses LINE to train embeddings for each epoch and then calculates approximate k-nearest neighbors for each word $v$ in embedding space. Suppose we have approximate k-nearest neighbors $a N N_{t}(v)$ and $a N N_{t+1}(v)$ of word $v$ from two epochs, we can evaluate the difference between the two sets through the Jaccard Distance:

$\operatorname{Score}(v)=\frac{\left|a N N_{t}(v) \cup a N N_{t+1}(v)\right|-\left|a N N_{t}(v) \bigcap a N N_{t+1}(v)\right|}{\left|a N N_{t}(v) \bigcup a N N_{t+1}(v)\right|}$.

The difference between aNN+Jaccard and our proposed method, denoted as aNN+Bayesian, is that the former does not use Bayesian surprise to measure the shift.

Actually, some heuristics are general and can be combined. For example, one can always initialize the embeddings of the current epoch with the embeddings of the previous epoch. One can also use other embedding learning algorithms (such as Word2Vec) instead of LINE. This creates more baselines such as LINE+Init+aNN+Jaccard or even variations of the proposed method such as LINE+Init+ aNN+Bayesian. We explore several combinations in Table 1.

\subsection{Data Sets}

We choose four large-scale, real-world data sets, ACM Abstract [38], DBLP Abstract [38], ACMDL Papers [8] and Google Books Ngrams [28]. These data sets represent formal academic writings and professional books, which are commonly used in existing studies to analyze semantic shifts $[14,21,41]$.

\section{ACM Abstract}

The ACM Abstract dataset consists of more than 2.38 million papers, 1.67 million of which have abstracts ${ }^{3}$. It spans 66 years from 1951 to 2016 . We divide the corpus into 66 snapshots.

\section{DBLP Abstract}

The DBLP Abstract dataset consists of more than 3.07 million papers, 2.55 million of which have abstracts ${ }^{4}$. It spans 64 years from 1954 to 2017 . We divide the corpus into 64 snapshots.

\section{ACMDL}

The ACMDL dataset consists of full text papers of ACM publications. It contains nearly 197 thousand conference papers and nearly 77 thousand journal papers. This data spans 61 years from 1951 to 2011. We divide the corpus into 61 separate snapshots.

\section{The Google Books Ngram Dataset}

The Google Books Ngram dataset ${ }^{5}$ consists of more than 5 million digitized books in eight languages over five countries and nearly $10^{10}$ observed words, in forms of n-grams $(n \in[1,5])$ with term frequency and document frequency. Following the sampling procedure described in [21], we sample 200 million 5-grams from English Fictions and consider the years from 1800 to 2004 . We divide the data into 41 snapshots by every 5 years.

\subsection{Ground Truth}

To quantitatively evaluate the performance of proposed method and the competing methods, we will need the ground truth of words that have truly changed their meanings. Unfortunately, such ground-truth is hard to obtain and does not exist on all data sets.

\footnotetext{
${ }^{3}$ This data is from: https://aminer.org/citation, we use ACM-V8 in our experiment. ${ }^{4}$ This data is from: https://aminer.org/citation, we use DBLP-V10 in our experiment. ${ }^{5}$ This data is available at: http://storage.googleapis.com/books/ngrams/books/datasetsv2.html
} 
Table 1: Performance of competing methods: LINE+Init+aNN+Bayesian achieves top performance.

\begin{tabular}{|c|c|c|c|c|c|c|c|c|}
\hline & \multicolumn{2}{|c|}{ ACM Abstract Dataset } & \multicolumn{2}{|c|}{ DBLP Abstract Dataset } & \multicolumn{2}{|c|}{ ACMDL Dataset } & \multicolumn{2}{|c|}{ Google Books Dataset } \\
\hline Method & Recall & MAP & Recall & MAP & Recall & MAP & Recall & MAP \\
\hline LINE+Init & 98.75 & $62.44_{\diamond}^{\star \star \star}$ & 99.75 & 65.01 & 98.25 & $60.52 \star \star \star$ & 75.00 & 51.23 \\
\hline word2vec+LLR & $19.75_{\infty \diamond \star \Delta \star}^{\star \star}$ & $6.99_{\infty \infty \star}^{\star \star \star}$ & $23.50_{\infty \star \Delta}^{\star \star \star}$ & $5.26_{\infty \infty \star}^{\star \star}$ & $17.50_{\diamond \star \infty}^{\star \star}$ & $5.04_{\Delta \infty \diamond}^{\star \star}$ & $4.50_{\Delta \infty \star}^{\star \star}$ & $0.48_{\infty \infty \star}^{\star \star \star}$ \\
\hline word2vec+LR & $39.75_{\Delta \infty \star}^{\star \star}$ & $18.78_{\infty \infty \diamond}^{\star \star}$ & $48.50_{\infty \star \star \star}^{\star \star}$ & $22.47_{\infty \star \infty \star}^{\star \star}$ & $58.75_{\Delta \infty \star}^{\star \star}$ & $27.49_{\infty \diamond \star \star}^{\star \star}$ & $31.75_{\infty \star \infty}^{\star \star}$ & $14.88_{\infty \star \Delta}^{\star \star}$ \\
\hline LINE+LLR & $57.00_{\Delta \infty \star}^{\star \star}$ & $21.80_{\diamond \diamond \diamond}^{\star \star \star}$ & $69.00_{\infty \infty \star \infty \star}^{\star \star}$ & $31.89_{\Delta \infty \star}^{\star \star \star}$ & $4.25_{\Delta \diamond \diamond \star}^{\star \star}$ & $1.83_{\Delta \infty \star}^{\star \star}$ & $57.25_{\infty \star \infty}^{\star \star}$ & $27.77_{\Delta \star \Delta}^{\star \star}$ \\
\hline $\mathrm{LINE}+\mathrm{LR}$ & 96.75 & $52.90 \diamond$ & 98.75 & $58.64 \diamond$ & $91.25_{\diamond}^{\star}$ & 48.72000 & 72.50 & $42.35 \bowtie \infty$ \\
\hline LINE+Init+LLR & $77.00_{\infty \diamond \star \star \star}^{\star}$ & $36.02_{\infty \infty \diamond}^{\star \star}$ & $80.00_{\infty \diamond \star \star \star}^{\star \star \star}$ & $39.66_{\infty \infty \star}^{\star \star \star}$ & $8.75_{\infty \infty \star}^{\star \star}$ & $2.40_{\diamond \star \Delta}^{\star \star}$ & $63.25_{\infty \infty \infty}^{\star}$ & 34.11000 \\
\hline LINE+Init+LR & 98.00 & 58.34 & 99.75 & 64.89 & 97.75 & 57.43 & 76.00 & 52.31 \\
\hline LINE+aNN+Jaccard & $93.50_{\diamond}^{\star}$ & $47.38_{\infty \infty}^{\star \star}$ & $85.75_{\diamond}^{\star}$ & $48.78_{\infty}^{\star \star}$ & $79.00_{\infty \infty \star}^{\star \star \star}$ & $32.61_{\Delta \infty \star}^{\star \star \star}$ & $68.00_{\diamond}^{\star}$ & 37.6300 \\
\hline LINE+Init+aNN+Jaccard & 97.00 & 54.51 & 98.75 & 60.47 & 95.50 & 49.50 & 73.00 & 44.69 \\
\hline Proposed methods & & & & & & & & \\
\hline LINE+aNN+Bayesian & $92.50_{\diamond}^{\star}$ & $87.54_{\infty \infty \diamond}^{\star \star}$ & $98.00 \diamond$ & $96.70_{\infty \infty \star}^{\star \star \star}$ & $94.50 \diamond$ & $89.41_{\Delta \infty \star \Delta \star}^{\star \star}$ & $96.50_{\infty \infty \star \star \star}^{\star \star}$ & 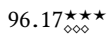 \\
\hline LINE+Init+aNN+Bayesian & $94.25 \diamond$ & $91.82_{\infty \infty \star \star \star}^{\star \star}$ & 98.00 & 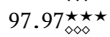 & $97.75 \star$ & $93.98_{0 \infty \star \star \star}^{\star \star}$ & $99.00_{0 \infty \star \star \star}^{\star \star}$ & 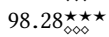 \\
\hline
\end{tabular}

All numbers are in percentage. “ $\star(\star \star, \star \star \star)$ " indicates the result is significant over LINE+Init+aNN+faccard (in general the best among all non-Bayesian, manifold-based models) according to paired t-test [42] at level $0.05(0.01,0.001)$. " $\diamond(\diamond \diamond, \infty \diamond \diamond)$ " indicates the result is significant over LINE+Init $+L R$ according to paired $\mathrm{t}$-test at level $0.05(0.01,0.001)$.

Kulkarni el at. [21] created a reference list $R$ of 21 words that have changed meanings with a very high probability during the time frame of the Google book data, which has been used as a benchmark [12, 18, 20, 41]. We adopt the same evaluation procedure and compared the competing methods.

Each algorithm outputs a ranked list of words based on their scores or ranks of possible shifts in any 5-year period on the Google books data set. Denote such a ranked list by $L$, we evaluate the Precision@p with respect to the reference list $R$, where $p$ is the number of top words for evaluation in the result list $L$.

We plot the 5 representative methods in Figure 1. From Figure 1, we can see that our proposed algorithm LINE+Init+ann+Bayesian can effectively identify shifts of word semantics, which clearly outperforms other baselines in most of the cases.

Note that in this test scenario, even the precision of the best method does not exceed $40 \%$. This is not surprising as the 21 words is by no means a comprehensive set of words with semantic changes. These methods may have correctly identified other words, but they are just not included in the small reference list. We show some of these words found by our methods in Section 6.2.

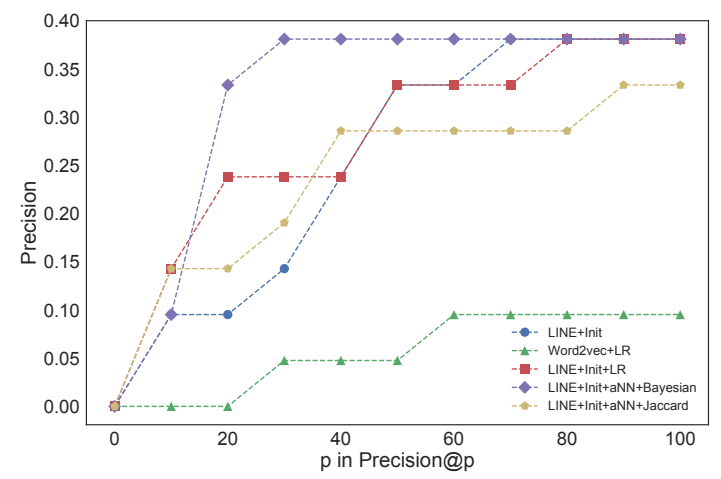

Figure 1: Performance on identifying ground truth.

\subsection{Synthetic Ground Truth}

The reference list $R$ of 21 words is insufficient to obtain statistical significance, and such a list does not exist for other data sets. To better compare the methods, Kulkarni et al. [21] proposed a neat procedure. They created a synthetic ground truth to a data set in which real words are injected with clear linguistic shifts.

Following their procedure, we create a synthetic ground truth for each data set. We randomly sample 200 pairs of words with similar frequency from certain time periods (in Google Books 19502000, in ACMDL 1991-2010, in DBLP abstract 1991-2016, in ACM abstract 1991-2015) with two conditions: 1) their frequencies in the entire data set are larger than 2,000, and 2) their frequencies in the current epoch and several previous epochs are larger than 200. The two restrictions are set to ensure that the words being selected are relatively stable and less noisy.

After obtaining the pairs of words, we artificially swap the paired words in a randomly selected time period. In this way, we know that the meanings of these words have significantly changed in the corresponding time period. Naturally, a good algorithm should be able to identify such words in that period and and rank them high.

To compare the performance of different models, in each period we require each model to rank 50 words by the score of semantic shifts. Based on the synthetic ground truth, we calculate the Recall and the Mean Average Precision (MAP) of each method.

The overall performance of all competing models are shown in Table 1, it is clear that our proposed methods LINE+aNN+Bayesian and LINE+Init+aNN+Bayesian both outperform all the other methods in MAP, without a sacrifice of Recall. LINE+Init+aNN+Bayesian achieves near-perfect MAP and Recall on all data sets, while the best performing competing methods obtain a MAP lower than $80 \%$.

Comparing between the word embedding methods, the methods using LINE+Init to learn the embeddings perform better than the methods using LINE alone or using Word2Vec, even if the vectors are aligned later or a semantic manifold is built. This is possibly due to the fact that using last period's embedding to initialize the current period not only aligns the embedding space but also reduces noises in a single epoch. In addition, models using LINE perform better than models using Word2Vec. 


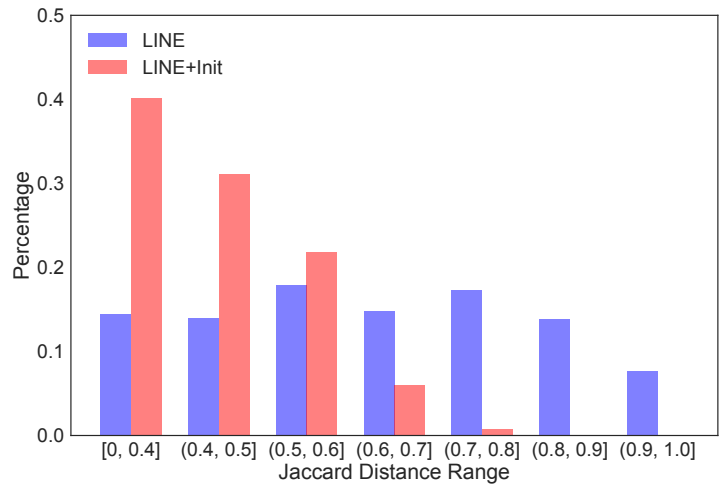

(a) Percentage of each Jaccard Distance interval

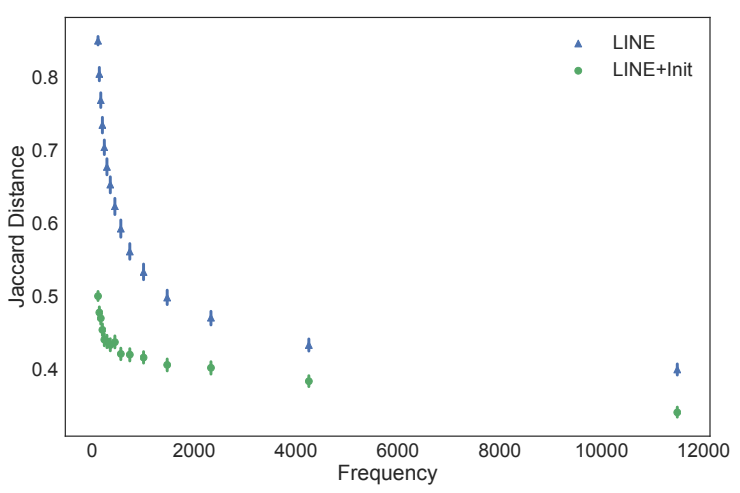

(b) Average Jaccard Distance of frequency

Figure 2: The Jaccard Distance distribution of two embedding coordinate systems trained with same parameters independently.

Comparing different versions of alignment-based models, we find that the Linear Regression model performs better than Local Linear Regression model in general. The reason might once again be the existence of a large number of noisy points.

It is a little surprising that applying initialization before training embedding also improves the performance of manifold-based methods, as a topological structure does not need the alignment of embeddings. Again, our hypothesis is that a good initialization of embedding learning not only aligns the embedding space but also reduces noise and uncertainty in a single epoch.

Comparing methods that use semantic manifolds, we can see a significant improvement introduced by Bayesian surprise. Comparing to Jaccard distance, Bayesian surprise takes all the historical nearest neighbors into consideration and is robust to uncertainty and noise. When Bayesian surprise is used, the difference between using Init+ or not (4.57\% in MAP on ACMDL) is much smaller than when Jaccard is used (16.89\% in MAP on ACMDL). This supports our hypothesis that initializing word embeddings using previous epochs is reducing the noise and uncertainty in a single epoch More exploration of this phenomenon is deferred to Section 6.1.

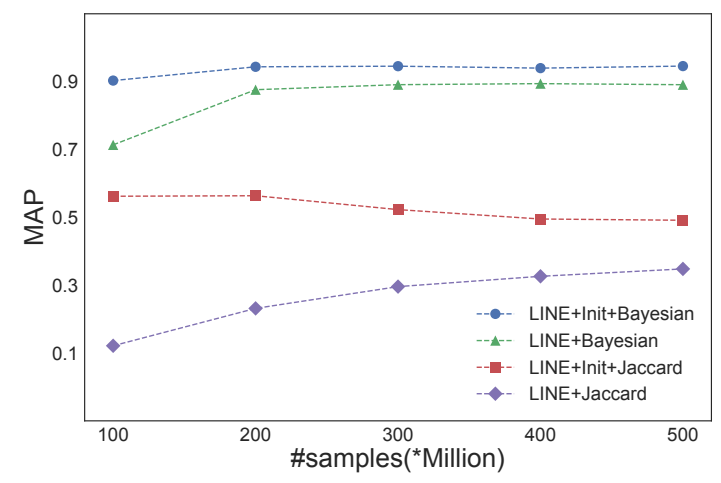

Figure 3: Performance of using different number of training samples in Synthetic ACMDL

\section{DISCUSSIONS}

The overall results show that our proposed methods clearly outperform baselines on detecting words with semantic shifts. Let us turn our attention to some specific behaviors of the models.

\subsection{The Influence of Noisy Points}

In Section 5.4, we find that nearly all models trained with Init+ perform better than those with random initialization of embeddings. This also holds for manifold-based models, which in theory should not depend on the initialization. We hypothesize that this is because Init+ can help us reduce noisy points, which are words that appear infrequently and likely generate false positives. In order to verify our hypothesis, we first find a way to quantify the stability of embedding models with and without Init+.

We focus on two methods: LINE and LINE+Init, which only differ by the initialization. We train both models twice, and then measure the dissimilarity of the nearest neighbors of every word between the first and the second run. Intuitively, if a model is stable enough, the neighbors of a word in two runs should not change much. From Figure 2(a), we can see that LINE+Init has more words with smaller dissimilarity of neighbors, measured by the Jaccard Distance. This indicates that Init+ indeed improves the stability of a model. Figure 2(b) shows that words with low frequency are more likely to have a high dissimilarity of neighbors between two runs. These infrequent words could easily produce noises for models without Init+. Thus a general suggestion is to adopt Init+. Another solution could be to filter out infrequent words beforehand.

Another possible way to reduce noise is trying to train embeddings sufficiently. To verify this, we vary the hyper-parameter "samples" in the LINE model, which controls the number of examples for training. We show the results on Synthetic ACMDL data set, as the same conclusion is observed on all four data sets. As Figure 3 shows, as samples increase, the advantage of models with Init+ shrinks. This empirically proves that with more sufficient training, we are less likely to suffer from the noises. 
Table 2: Sample of words detected by LINE+Init+ann+Bayesian on four data sets.

\begin{tabular}{c|c|c|c|c|c|c} 
& Word & rank & year & Past neighbors & Present neighbors \\
\hline ACM Abstract & cloud & 1 & 2009 & surface, volumetric, terrain, subsurface, fiduciary & grid, ubiquitious, percasive, desktop, compute \\
ACM Abstract & deep & 1 & 2014 & deeper, insight, understand, dig, deeply & clouds, grid, computing, virtualized, ubiquitous \\
\hline DBLP Abstract & cloud & 1 & 2009 & clouds, surface, brdf, ice, subsurface & multilayer, neural, spiking, multilayered, recurrent \\
DBLP Abstract & deep & 1 & 2013 & deeper, propagation, ld, shallow, integration & apache, hadoop, mapreduce, opencl, standalone \\
DBLP Abstract & spark & 1 & 2015 & php, engine, perl, commercial, labview & android, apps, smartphone, microsoft \\
DBLP Abstract & app & 5 & 2011 & viterbi, ml, sam, turbo, me & daemon, host, linux, unix, servlet \\
DBLP Abstract & pooling & 8 & 2015 & combing, weighting, grouping, fusion, pyramid & hosting, services, enterprise, infrastructure, virtualization \\
\hline ACMDL & guest & 5 & 2003 & editor, editors, writer, newsletter, presenter & ware, tracking, dynamic, hard, modeling \\
ACMDL & cloud & 9 & 2009 & clouds, earth, map, sphere, terrain & phone, mobile, telephone, wallet, card \\
ACMDL & soft & 20 & 1991 & practical, on, pushed, turned, highlighted & lesbian, lesbians, urban, homosexual, adults \\
Google Books & cell & 1 & 2000 & cabin, tent, cubicle, locker, trailer & site, website, releases, internet, quest \\
Google Books & gay & 1 & 1985 & lively, cheerful, courteous, sensitive, prosperous & maze, galaxy, peninsula, network, northern &
\end{tabular}

\subsection{What Our Method Learns}

To gain a better understanding of the proposed method, we qualitatively analyzed some words to which new meanings are attached.

Table 2 shows results from our LINE+Init+ann+Bayesian method on four data sets. Some words are singled out because that they have truly acquired new meanings. For example, the word cloud acquired a new sense "networks of computing equipment" in 2009 due to the development of cloud service. The same thing happens to words web, gay, cell and guest.

Interestingly, another type of words found out by our model have not necessarily changed meaning on its own, but are serving as a part of a new concept. For example, the word deep, together with word learning, constitutes a new concept in Computer Science. Similar things happened for words soft, spark and pooling.

In Figures 4 and 5, we present two visualizations of how certain words have shifted in the semantic manifolds. The shifts clearly present differences in the local neighborhoods, which are captured by Bayesian surprise.

\section{CONCLUSIONS AND FUTURE WORK}

We have introduced a novel method to detect shifts of word semantics over time. Our method represents word semantics in a manifold space, which is robust to distortions and does not require alignments of embeddings. Bayesian surprise is applied to detect changes in the topological space, which significantly outperforms a complete set of baselines on four real world data sets with both real and synthetic ground truth. Through the experiments, we also obtained a comprehensive comparison among all existing methods.

One future direction of this work will focus on analyzing which particular new meanings are added to the words. Moreover, generalizing our method to other network tasks is also a promising direction, e.g., detecting a user's interest change in social network enables us to better recommend new topics and new friends.

\section{Acknowledgment}

This work was supported in part by the National Science Foundation under grant numbers 1054199, 1633370, 1131500 and 1620319, and in part by the NSFC(61625107), and Key program of Zhejiang Province (2015C01027).

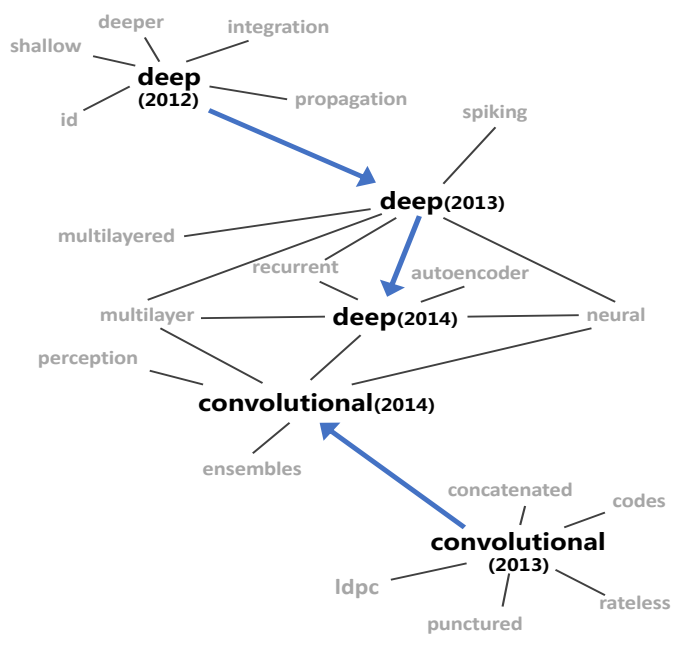

Figure 4: Shifts of "deep" and "convolutional" in the semantic manifold (DBLP Abstract).

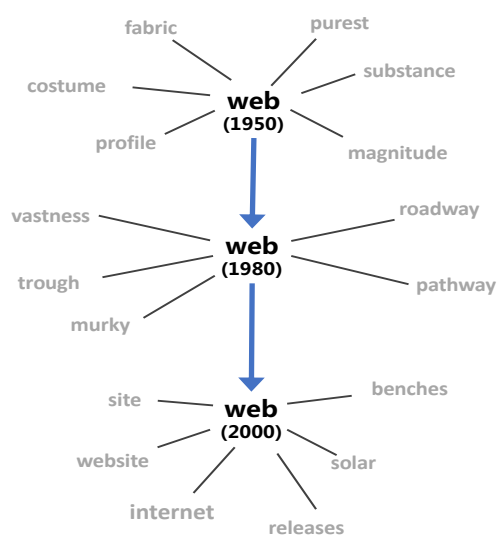

Figure 5: A shift of the word "web" in the semantic manifold (Google Books). 


\section{REFERENCES}

[1] [n. d.]. Preemptive. https://en.wikipedia.org/wiki/Bayes\%27 theorem.

[2] [n. d.]. Preemptive. https://en.wikipedia.org/wiki/Jaccard_index.

[3] Pierre Baldi and Laurent Itti. 2010. Of Bits and Wows: a Bayesian Theory of Surprise With Applications to Attention. Neural Networks (2010).

[4] Yoshua Bengio, Réjean Ducharme, Pascal Vincent, and Christian Jauvin. 2003. A Neural Probabilistic Language Model. Journal of machine learning research (2003).

[5] Andreas Blank. 1999. Why do new meanings occur? A cognitive typology of the motivations for lexical semantic change. Historical semantics and cognition 13 (1999), 6.

[6] David M Blei and John D Lafferty. 2006. Dynamic topic models. In Proceedings of the 23rd international conference on Machine learning. ACM, 113-120.

[7] Igor Brigadir, Derek Greene, and Pádraig Cunningham. 2015. Analyzing Discourse Communities With Distributional Semantic Models. In Proceedings of the ACM Web Science Conference. 27.

[8] Yinlin Chen and Edward A Fox. 2014. Using ACM DL paper metadata as an auxiliary source for building educational collections. In Proceedings of the 14th ACM/IEEE-CS foint Conference on Digital Libraries. IEEE Press, 137-140.

[9] Kenneth Ward Church and Patrick Hanks. 1990. Word Association Norms, Mutual Information, and Lexicography. Computational linguistics (1990).

[10] Lea Frermann and Mirella Lapata. 2016. A Bayesian Model of Diachronic Meaning Change. Transactions of the Association for Computational Linguistics (2016).

[11] Ioannis Gkioulekas, Georgios Evangelopoulos, and Petros Maragos. 2010. Spatial Bayesian surprise for image saliency and quality assessment. In Image Processing (ICIP), 2010 17th IEEE International Conference on. IEEE, 1081-1084.

[12] Kristina Gulordava and Marco Baroni. 2011. A Distributional Similarity Approach to The Detection of Semantic Change in The Google Books Ngram Corpus. In Proceedings of the GEMS 2011 Workshop on GEometrical Models of Natural Language Semantics. 67-71.

[13] William L Hamilton, Jure Leskovec, and Dan Jurafsky. 2016. Cultural Shift or Linguistic Drift? Comparing Two Computational Measures of Semantic Change. In Proceedings of the Conference on Empirical Methods in Natural Language Processing. Conference on Empirical Methods in Natural Language Processing, Vol. 2016 2116.

[14] William L Hamilton, Jure Leskovec, and Dan Jurafsky. 2016. Diachronic Word Embeddings Reveal Statistical Laws of Semantic Change. In Proceedings of the 54th Annual Meeting of the Association for Computational Linguistics (Volume 1: Long Papers), Vol. 1. 1489-1501.

[15] Barbara S Held and Edward Pols. 1985. The confusion about epistemology and âĂIJepistemologyâĂÎâĂTand what to do about it. Family process 24, 4 (1985) 509-517.

[16] Laurent Itti and Pierre Baldi. 2009. Bayesian Surprise Attracts Human Attention. Vision research (2009).

[17] Laurent Itti and Pierre F Baldi. 2006. Bayesian surprise attracts human attention. In Advances in neural information processing systems. 547-554.

[18] Adam Jatowt and Kevin Duh. 2014. A Framework for Analyzing Semantic Change of Words Across Time. In Proceedings of the 14th ACM/IEEE-CS Joint Conference on Digital Libraries. 229-238.

[19] Margarita Kay. 1979. Lexemic change and semantic shift in disease names. Culture, medicine and psychiatry 3, 1 (1979), 73-94.

[20] Yoon Kim, Yi-I Chiu, Kentaro Hanaki, Darshan Hegde, and Slav Petrov. 2014 Temporal Analysis of Language through Neural Language Models. ACL 2014 (2014), 61.

[21] Vivek Kulkarni, Rami Al-Rfou, Bryan Perozzi, and Steven Skiena. 2015. Statistically Significant Detection of Linguistic Change. In Proceedings of the 24th International Conference on World Wide Web. 625-635.

[22] Solomon Kullback. 1997. Information Theory and Statistics. Courier Corporation.
[23] John Lee. 2010. Introduction to topological manifolds. Vol. 940. Springer Science \& Business Media.

[24] Omer Levy, Yoav Goldberg, and Ido Dagan. 2015. Improving Distributional Similarity With Lessons Learned From Word Embeddings. Transactions of the Association for Computational Linguistics (2015).

[25] Annie P Louis. 2014. A Bayesian Method to Incorporate Background Knowledge During Automatic Text Summarization. Association for Computational Linguistics.

[26] Aloysius P Martinich. 1984. Communication and reference. Walter de Gruyter.

[27] Qiaozhu Mei and ChengXiang Zhai. 2005. Discovering evolutionary theme patterns from text: an exploration of temporal text mining. In Proceedings of the eleventh ACM SIGKDD international conference on Knowledge discovery in data mining. ACM, 198-207.

[28] Jean-Baptiste Michel, Yuan Kui Shen, Aviva Presser Aiden, Adrian Veres, Matthew K Gray, Joseph P Pickett, Dale Hoiberg, Dan Clancy, Peter Norvig, Jon Orwant, et al. 2011. Quantitative analysis of culture using millions of digitized books. science 331, 6014 (2011), 176-182.

[29] Tomas Mikolov, Kai Chen, Greg Corrado, and Jeffrey Dean. 2013. Efficient Estimation of Word Representations in Vector Space. arXiv preprint arXiv:1301.3781 mation

[30] Tomas Mikolov, Ilya Sutskever, Kai Chen, Greg S Corrado, and Jeff Dean. 2013. Distributed Representations of Words and Phrases and Their Compositionality. In Advances in neural information processing systems. 3111-3119.

[31] Dirk Ostwald, Bernhard Spitzer, Matthias Guggenmos, Timo T Schmidt, Stefan J Kiebel, and Felix Blankenburg. 2012. Evidence for neural encoding of Bayesian surprise in human somatosensation. Neuroimage 62, 1 (2012), 177-188.

[32] William D Penny. 2001. Kullback-liebler Divergences of Normal, Gamma, Dirichlet and Wishart densities. Wellcome Department of Cognitive Neurology (2001).

[33] Bryan Perozzi, Rami Al-Rfou, and Steven Skiena. 2014. Deepwalk: Online Learning of Social Representations. In Proceedings of the 20th ACM SIGKDD international conference on Knowledge discovery and data mining. 701-710.

[34] Ananth Ranganathan and Frank Dellaert. 2009. Bayesian surprise and landmark detection. In Robotics and Automation, 2009. ICRA'09. IEEE International Conference on. IEEE, 2017-2023.

[35] Benjamin Recht, Christopher Re, Stephen Wright, and Feng Niu. 2011. Hogwild: A Lock-free Approach to Parallelizing Stochastic Gradient Descent. In Advances in neural information processing systems. 693-701.

[36] Jian Tang, Jingzhou Liu, Ming Zhang, and Qiaozhu Mei. 2016. Visualizing Largescale and High-dimensional Data. In Proceedings of the 25th International Conference on World Wide Web. 287-297.

[37] Jian Tang, Meng Qu, Mingzhe Wang, Ming Zhang, Jun Yan, and Qiaozhu Mei. 2015. LINE: Large-scale Information Network Embedding. In Proceedings of the 24th International Conference on World Wide Web. 1067-1077.

[38] Jie Tang, Jing Zhang, Limin Yao, Juanzi Li, Li Zhang, and Zhong Su. 2008. Arnetminer: extraction and mining of academic social networks. In Proceedings of the 14th ACM SIGKDD international conference on Knowledge discovery and data mining. ACM, 990-998.

[39] Elizabeth Closs Traugott. 1989. On the rise of epistemic meanings in English: An example of subjectification in semantic change. Language (1989), 31-55.

[40] Xuerui Wang and Andrew McCallum. 2006. Topics over time: a non-Markov continuous-time model of topical trends. In Proceedings of the 12th ACM SIGKDD international conference on Knowledge discovery and data mining. ACM, 424-433.

[41] Derry Tanti Wijaya and Reyyan Yeniterzi. 2011. Understanding Semantic Change of Words Over Centuries. In Proceedings of the 2011 international workshop on DETecting and Exploiting Cultural diversiTy on the social web. 35-40.

[42] Yiming Yang and Xin Liu. 1999. A re-examination of text categorization methods. In Proceedings of the 22nd annual international ACM SIGIR conference on Research and development in information retrieval. ACM, 42-49. 